Article

\title{
Fede cristiana, devozione mariana e pandemia da covid-19. II"caso" della comunità di Biancavilla (Ct)
}

\author{
VALERIO CIAROCCHI ${ }^{1}$
}

\begin{abstract}
Riassunto. La crisi drammaticamente scaturita dall'improvvisa pandemia per il covid-19 ha avuto conseguenze immediate e di lungo termine. Dal punto di vista religioso, quali sono state le risposte? La comunità civile ed ecclesiale biancavillese è un unicum nella provincia etnea e nel Valdemone, poiché è l'unica d'origine arbëreshë, pur ormai latinizzata, giunta ed infine stanziatasi alle falde dell'Etna, dopo l'esilio seguito alla caduta di Bisanzio. Gli esuli portarono con sé una icona di Maria dell'Elemosina, che è in qualche misura allorigine della fondazione dell'abitato. Al culto, prima bizantino, poi latino, della Vergine della Misericordia, e della sua icona, è legata la vicenda storica, sociale e religiosa locale. Ad essa i biancavillesi hanno sempre rivolto la loro devozione, tanto più in occasione di guerre, carestie, pestilenze, ottenendo, secondo la fede del popolo, il favore della Vergine, nonostante le comunità vicine venissero ugualmente colpite. Vogliamo riportare il "caso" della comunità di Biancavilla, che ha affrontato l'emergenza sanitaria da covid-19, anche dal punto di vista religioso con un costante, ampio, vario impegno, non solo liturgico o devozionale, ma anche educativo. Esprimendo ancora una volta la propria fede cristiana nella devozione alla Vergine dell'Elemosina, sua patrona. Mentre non è mancato l'impegno del clero per una costante attività liturgica e pastorale, parimenti i fedeli hanno mantenuto le attività oratoriali ed educative attraverso l'uso della rete internet, servendosi di tutti i canali di comunicazione a disposizione, con risultati positivi anche rispetto ad altre comunità civili e religiose, durante il lungo periodo d'isolamento, mantenendo vivi i rapporti personali e comunitari. La devozione mariana biancavillese ha dunque permeato e fatto da agglutinante della tenuta complessiva della cittadina di Biancavilla.
\end{abstract}

Parole chiave: covid-19, devozione, fede, formazione, comunità.

\begin{abstract}
The crisis dramatically resulting from the sudden pandemic for Covid-19 had immediate and long-term consequences. From a religious point of view, what were the answers? The civil and ecclesial community of Biancavilla country is unique in the Etna province and in Valdemone, since it is the only one of Arbëreshë origin, although now Latinized, arrived and finally settled at the foot of Etna, after the exile following the fall of Byzantium. The exiles brought with them an icon of the Mary of almsgiving, which is to some extent at the origin of the foundation of the town.
\end{abstract}

\footnotetext{
${ }^{1}$ Docente Invitato di Musicologia Liturgica e Catechetica presso l'Istituto Teologico "San Tommaso d'Aquino" di Messina, aggregato alla Facoltà di Teologia dell'Università Pontificia Salesiana di Roma. Coordinatore sezione "arte, musica e catechetica" presso il Centro di Pedagogia Religiosa "G. Cravotta", operante presso il medesimo Istituto. Per l'A.A. 2019-2020 docente a contratto di musicologia e storia della musica, presso il Dipartimento di Civiltà Antiche e Moderne dell'Università degli Studi di Messina, nell'ambito del Master di I livello in "Civiltà e turismo religioso nel Sud-Italia. Per un modello operativo euro-mediterraneo”. Email: valeriociarocchi@yahoo.it.
} 
The local historical, social and religious history is linked to the cult, first Byzantine, then Latin, of the Virgin of Mercy, and its icon. The people of Biancavilla country have always devoted their devotion to it, especially on the occasion of wars, famines, plagues, obtaining, according to the faith of the people, the favor of the Virgin, despite the fact that neighboring communities were equally affected. We want to report the "case" of the Biancavilla community, which faced the health emergency caused by Covid-19, also from a religious point of view with a constant, broad, varied commitment, not only liturgical or devotional, but also educational. Expressing once again its Christian faith in devotion to the Virgin of Alms, her patron. While the commitment of the clergy to a constant liturgical and pastoral activity has not been lacking, the faithful also maintained the oratorio and educational activities through the use of the internet, using all the communication channels available, with positive results also with respect to other civil and religious communities, during the long period of isolation, keeping personal and community relationships alive. The biancavillese marian devotion therefore permeated and acted as an agglutinant of the overall estate of the small town of Biancavilla.

Keywords: covid-19, devotion, faith, training, community.

\section{Introduzione}

La crisi drammaticamente scaturita dall'improvvisa pandemia per il covid-19, a cui abbiamo assistito nei primi mesi del 2020, ha avuto conseguenze immediate e di lungo termine. Essa ha messo a nudo il senso d'impotenza dell'uomo dinanzi ad un evento di portata mondiale. Ha messo alla prova la tenuta dei sistemi sanitari nazionali, della società stessa chiamata a misure restrittive importanti ed alle quali nessuno era pronto. Tuttavia, una crisi del genere porta inevitabilmente a fare i conti con tanti aspetti della vita e delle priorità. Essa ha investito direttamente tutto e tutti. Dal punto di vista religioso, quali sono state le risposte? In quale atteggiamento si è messa la fede di ciascuno, la devozione popolare delle comunità? Con quale armonizzazione rispetto alle indicazioni ufficiali dell'autorità ecclesiastica?

Noi intendiamo fare qui riferimento ad un'esperienza ben precisa. Essa dice una parte soltanto del tutto che è la Chiesa, intesa come comunità più ampia. Tuttavia caratteristica e significativa. Vogliamo qui riportare, osservare, descrivere il "caso" di una comunità in particolare rispetto ad un approccio più generale, intesa come cittadina e religiosa. La comunità ecclesiale di Biancavilla, cittadina etnea, che ha affrontato l'emergenza sanitaria, oltre che per tutti gli altri aspetti, anche dal punto di vista religioso con un costante, ampio, vario impegno, non solo liturgico o devozionale, ma anche educativo della comunità biancavillese. Esprimendo ancora una volta la propria fede cristiana nella devozione alla Vergine dell'Elemosina, patrona della cittadina fin dalla fondazione dell'abitato e la cui icona che si venera nell'omonima basilica è ancor oggi l'elemento aggregante e caratterizzante dei biancavillesi, secondo la singolare caratteristica dell'essere, quella di Biancavilla, ${ }^{2}$ l'unica comunità di origine arbëreshë, pur ormai latinizzata, giunta ed infine stanziatasi

\footnotetext{
${ }^{2}$ Situata a trenta chilometri nordovest circa dal capoluogo Catania, sul versante sud occidentale dell'Etna, ad oltre cinquecento metri s.l.m. e con poco meno di venticinquemila abitanti. È insignita del titolo di Città.
} 
alle falde dell'Etna, dopo l'esilio seguito alla caduta di Bisanzio. Osserveremo dunque un "caso", secondo il tema proposto, che è già dentro un altro "caso" religioso, storico, antropologico e culturale, al quale previamente faremo un rapido ma opportuno riferimento.

\section{L'arrivo degli arbëreshë alle pendici dell'Etna}

Le campagne di conquista che l'impero ottomano promosse nel Quattrocento, volsero a consolidarne la presenza nell'area balcanica. Di particolare tenacia fu la resistenza degli albanesi, guidati da Scanderbeg, che tuttavia, nonostante le ripetute vittorie mai risolutive a favore dell'indipendenza, dovettero scegliere tra la sottomissione ai turchi con la conseguente islamizzazione o quanto meno una difficile convivenza, e la via dell'esilio. Quest'ultima opzione fu infine scelta dalla gran parte di questi cristiani che presero la via dell'Occidente, accolti dall'allora Regno di Napoli e di Sicilia e con la protezione pontificia, diffondendosi particolarmente in Calabria ed in Sicilia, dove a tutt'oggi le comunità locali degli arbëreshë sono raccolte in eparchia.

In Sicilia la gran parte di questi esuli, cristiani di rito orientale, si raccolse in quella zona interna alle spalle di Palermo che oggi racchiude alcune comunità locali, unite nell'Eparchia di Piana degli Albanesi. In Sicilia orientale si fermò un altro gruppo di arbëreshë, alle falde dell'Etna. Le ragioni che determinarono questa peculiarità dividono gli storici. Si ipotizza sostanzialmente che questo gruppo non potè raggiungere i connazionali perché già troppo numerosi per essere accolti tutti nello stesso territorio. Gli arbëreshë fermatisi in zona etnea fondarono una loro comunità, che aveva peculiarità tutte proprie rispetto alle vicine cittadine di rito latino e tradizione occidentale. Nel 1482 una colonia di profughi, ${ }^{3}$ composta da circa trenta famiglie, proveniente da Scutari e guidata da Cesare De Masi, ${ }^{4}$ sbarcò in Sicilia, portando con sé l'icona dell'Eleùsa, una reliquia del soldato martire d'Arabia, Zenone, ed una croce lignea orientale. Passando dalla Piana di Catania, con destinazione finale Palermo e da lì verso quella che fu detta Piana dei Greci, oggi Piana degli Albanesi, onde riunirsi con i connazionali ivi stanziatisi, il gruppo si fermò a riposare nel feudo di Callìcari o Poggio Rosso, allora di proprietà dei Moncada di Adernò. Il feudatario del luogo, Gian Tommaso Moncada, chiese ed ottenne dal Governo del Regno, onde sfrut-

\footnotetext{
${ }^{3}$ Certamente essi erano cristiani, qualche studioso propende per il rito ortodosso (Lossky), qualcun altro si orienta verso il rito cattolico bizantino (Bucolo). Probabilmente gli esuli orientali chiesero protezione alla Chiesa latina, forse senza ottenerla immediatamente. Le manipolazioni successive delle fonti probabilmente tradivano l'intenzione di dare legittimazione alle pratiche devozionali dei "greci" (in realtà più albanesi che ellenici, ma percepiti come tali perché celebravano in rito greco) agli occhi del clero e dei fedeli indigeni di rito latino. Piuttosto, secondo il Perroy, questi connazionali di Scanderbeg, lui sì cristiano ortodosso, erano un «ramo della famiglia illirica che avevano conservato la loro individualità $[\ldots]$ per custodire le proprie tradizioni religiose nazionali ed autocefale» (Perroy, 1958, pp. 474-475). A Biancavilla l'eco di queste ascendenze balcaniche si ritrova sia nella toponomastica (le vie Scutari, Cesare De Masi, Albania, Callicari, Castriota, Marco Botzaris) sia in alcuni cognomi (Bua, Bisicchia, Greco, Ingiulla, Papotto, Stissi), oltre che nel Circolo Castriota, tuttora esistente.

${ }^{4}$ Condottiero dell'esercito di Giorgio Castriota Scanderbeg. L'abilità militare ed il senso dell'onore e della fedeltà ne fecero delle milizie particolarmente richieste nel Regno di Napoli. Fino alla caduta del regno borbonico si segnalano reggimenti "Real Albania" e "Real Macedonia". D’altra parte va anche detto che queste comunità, autonome anche dal punto di vista difensivo, non furono sempre facilmente dominabili dai vari signorotti locali e questo, insieme agli altri elementi culturali gelosamente preservati e custoditi, permise la sopravvivenza delle tradizioni arbëreshë e del rito bizantino laddove le comunità rimasero vicine ed unite. Si legga per un primo approccio: Li Cauli, G. \& Melluso, L., (2015).
} 
tare al meglio il feudo con una manodopera volenterosa ed a basso costo, la licentia populandi e la stipula dei capitoli di fondazione, "veri e propri atti notarili che stabilivano diritti e doveri dei contraenti e che oggi costituiscono una documentazione assai importante al fine di valutare appieno le reali condizioni economiche e sociali e nelle quali nacquero e si svilupparono i nuovi insediamenti umani» (Monforte, 2008, p. 35) ${ }^{5}$ concedendo, a sua volta, agli arbëreshë il permesso legale di caseggiare per tre volte, fino alla definitiva concessione che portò alla vera e propria fondazione della cittadina. Gli eventi accaduti agli arbëreshë di Callìcari furono del tutto comuni a quelli occorsi ai loro connazionali.

Fin qui la storia. La tradizione vuole che, posto l'accampamento, gli arbëreshë appesero l'icona di Maria dell'Elemosina ad un albero di fico. Il mattino seguente gli esuli la trovarono aggrovigliata fra i rami del fico, cresciuti nottetempo, al punto che non fu possibile districarla. ${ }^{6}$ Levento fu interpretato come la volontà della Madre di Dio di rimanere in quel luogo, poi detto Casale dei Greci. ${ }^{7}$ I profughi arbëreshë non si fermarono a Callìcari "perché lo impose loro la Madonna, ma perché nella fede riconobbero ex post in una serie di circostanze storiche concrete (ad es. la terra concessa a condizioni particolarmente vantaggiose [...]) la sua benevolenza materna" (Scaccianoce, 2015, p. 18). Nel primigenio Casale dei Greci, tenuto conto della tradizione cristiana orientale degli arbëreshë, si celebrava in greco, con clero di rito bizantino, che rispondeva comunque all'autorità ecclesiastica latina catanese, sotto il controllo dell'Arcipretura di Adernò (Bucolo, 1953, p. 46; Monforte, 2008, p. 93). ${ }^{8}$ Di quella iniziale colonizzazione diventata, con il tempo, un misto di narrazione storica ed epica, rimase viva soltanto la devozione all'icona mariana. Da questo dato si ripartì, in un rinnovato slancio ecumenico e di dialogo religioso, a ridosso del Concilio Vaticano II.

\section{L'icona di Santa Maria dell'Elemosina: descrizione e culto}

Detta icona appartiene, secondo Maria Pia Di Dario Guida, al genere delle icone postbizantine, le cui vicende segnarono una significativa rinascita della cultura bizantina che seguì la caduta di Bisanzio nel 1453 e che definirono sia le personalità artistiche degli autori sia gli ambiti religiosi, politici ed iconografici di ciascun paese a cui gli iconografi facevano riferimento ed in cui operavano. ${ }^{9}$

\footnotetext{
${ }^{5}$ Cfr. Longhitano, (2006).

6 "Siamo dinanzi, infatti, ad un racconto che esprime per immagini una verità fattuale: quelle circostanze favorevoli, che indussero gli esuli a stanziarsi nei "campi belli" (traduzione di "Callìcari"), sono state per i nostri avi i segni certi di un dono della misericordia e della benevolenza di Dio (cosa cè di più dolce di un fico?)" (Scaccianoce, 2015, pp. 17-18). Mircea Eliade parlava, in casi come questo, di "mito di fondazione". Eliade, (1968). Cfr. Bertanza, (2013). Elemento che gli studiosi di storia patria biancavillese come E. Portal, A. Distefano e P. Bucolo accennano appena senza esprimere nessuna rilevante interpretazione critica in merito né dilungandosi più del necessario.

${ }^{7}$ Dalla fine del Cinquecento denominato Biancavilla, forse in onore della regina Bianca di Navarra o come riferisce il Bucolo perché è "il paese e la villa di Maria, la bianca Regina del Cielo; bianca per la sua verginità immacolata; Regina, per il suo potere sulle anime” (Bucolo, 1953, p. 20).

${ }^{8}$ Cfr. Archivio Storico Diocesano dell'Arcidiocesi di Catania, (1507-1509), f. 88. Gli arbëreshë di Callìcari ebbero il rito greco per più di un secolo, poi soppiantato dal rito latino. Sulla più ampia questione delle tensioni tra latini e bizantini, che portarono papa Gregorio XIII ad istituire nel 1573 la Congregatio pro reformatione Graecorum, preposta alla riforma per i greci e per gli albanesi d'Italia, si rimanda alla lettura di Rodriquez, 2013, pp. 217-250. ${ }^{9}$ Cfr. Di Dario Guida, (2013), pp. 9-55.
} 
Figura 1. Emanuele Di Giovanni, "Il prodigio del fico". Gli arbëreshë si fermano a Callicari (1952). Affresco nella parete destra del presbiterio della Basilica Collegiata Santuario "Maria SS. dell'Elemosina" Biancavilla $(\mathrm{Ct})$.

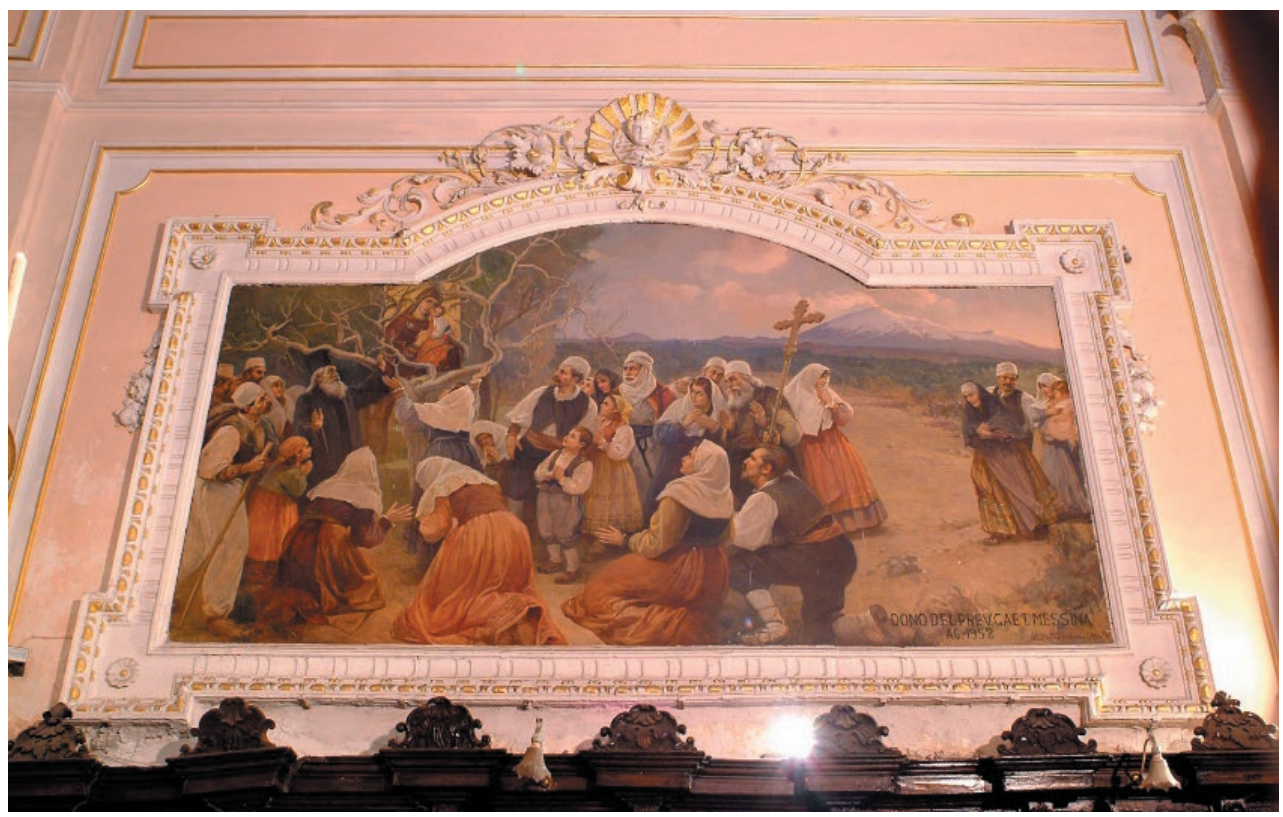

L'icona misura $67 \times 85 \mathrm{~cm}$., dipinta a tempera d'uovo su legno di cedro, databile agli inizi del XV secolo. È di netto stile greco e, nella parte mediana inferiore, richiama l'icona di Maria Santissima del Perpetuo Soccorso, alla quale, eccetto alcune differenze cromatiche, è sostanzialmente somigliante. L'icona porta tre stelle, ancorché scarsamente visibili, tipiche dell'iconografia mariana e simboleggianti la Verginità di Maria, prima, durante e dopo il parto. Nelle solennità l'icona viene rivestita della manta, o riza, in oro, argento e preziosi, che ne esalta i lineamenti con decori di gusto tardo-barocco. ${ }^{10}$ L'icona si presentava originariamente come una tavola a due ante pieghevoli nella loro linea mediana perpendicolare, probabilmente così concepita per essere più agevolmente trasportata come oggetto votivo. L'icona biancavillese, in sostanza, è stilisticamente una Madonna di scuola cretese. Appartiene al tipo dell'Eleousa: ${ }^{11}$ caratteristico di questo tipo è l'abbandono della rigidità della Odigitria per cedere il passo ad uno scambio affettuoso tra madre e bambino. L'Eleousa è dunque la Madre della Tenerezza.

\footnotetext{
${ }^{10}$ L'immagine è custodita nell'omonima Basilica Collegiata Santuario, nota anche come Chiesa Madre, in qualità di patrona principale di Biancavilla, nella quale sono anche venerati, come compatroni, San Placido e San Zenone, martiri. Larcivescovo di Catania, monsignor Guido Luigi Bentivoglio, elevò la Chiesa Madre a Santuario Mariano nel 1959; nel 1970 Paolo VI la fregiò del titolo e dignità di Basilica Minore Pontificia, con Bolla "In Sanctissimam Christi Matrem". Nel 2016, in occasione del Giubileo della Misericordia, la Chiesa Madre è stata dichiarata Santuario Giubilare.

${ }^{11}$ O Eleusa, da elemai, o eleoc, essere compassionevole, provare tenerezza. L'iconografo Tommaso Contarino ritiene che, riferita a questa icona, la migliore traduzione sia "del dolce bacio", ossia Glykophilousa.
} 
Figura 2. Icona di Maria SS. dell'Elemosina, Basilica Collegiata Santuario "Maria SS. dell'Elemosina" Biancavilla (Ct).

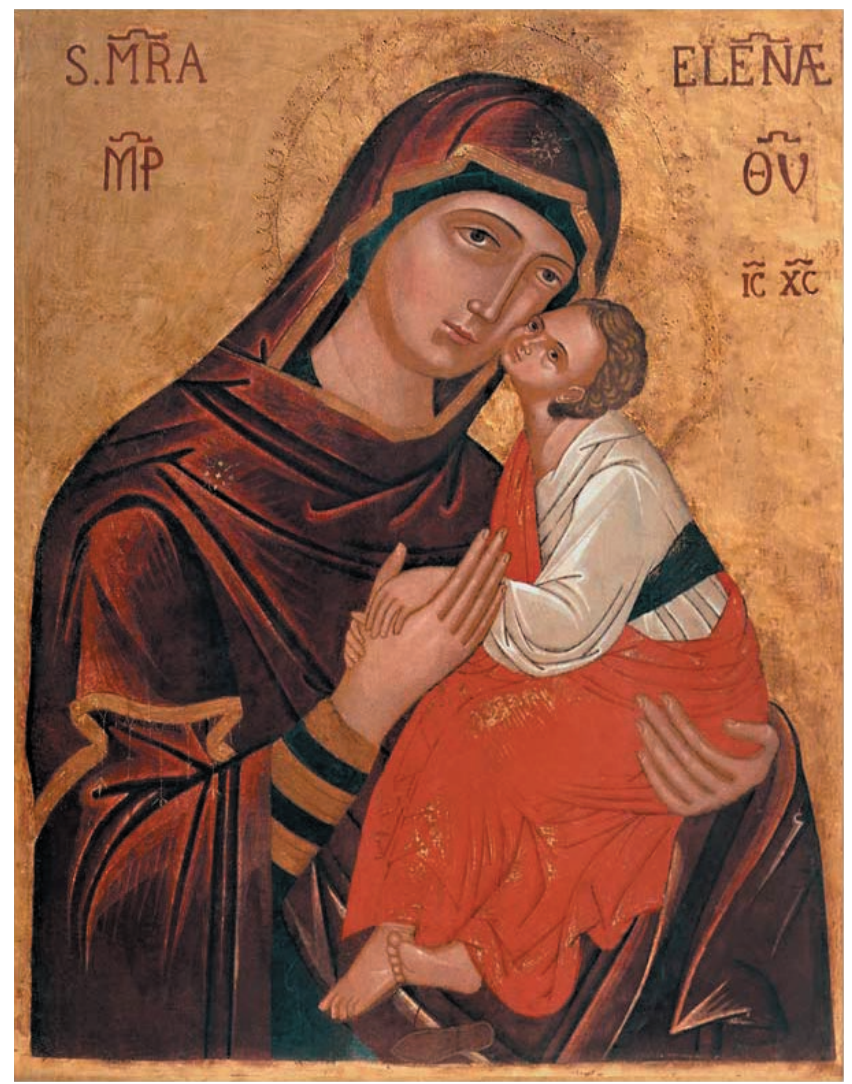

\section{Perché "Elemosina"?}

Per i biancavillesi, la "loro" icona è la Bedda Matri 'a Limosina, Mhthr Elehmosunh, Mater Elemosinae, variante di Mater Misericordiae, Madre della Misericordia visibile dell'invisibile Dio Misericordioso. ${ }^{12}$ Quasi istintivamente il popolo biancavillese sentiva ed avverte ancor oggi questa caratteristica della Madre di Dio. In Sicilia la Vergine è anzitutto la Bedda Matri, in una felice sintesi di bello e di buono, di kalokagathia, richiamo sempre vivo alla cultura ellenica della Magna Grecia, che si offre alla contemplazione dei fedeli: "L'icona è stata un simbolo per gli esuli e ha continuato ad esserlo per le generazioni che si sono susseguite a Biancavilla [...]. La nostra icona, dunque, è un simbolo per noi, in quanto ci ripresenta l'evento fondativo della nostra comunità. Pertanto essa costituisce un'evidenza storica simbolica, riferita ad un fatto, che rende presente e conoscibile, a noi

\footnotetext{
${ }^{12}$ Messa propria per la festa liturgica da celebrarsi il dieci ottobre. Cfr. Giovanni Paolo II, Decreto per l'istituzione della festa liturgica di S. Maria dell'Elemosina, 31 luglio 1981, in BASP, Cat. IV, Classe I, Fasc. II, n. 67.
} 
Figura 3. Biancavilla (Ct), Basilica Collegiata Santuario "Maria SS. dell'Elemosina" e piazza Roma.

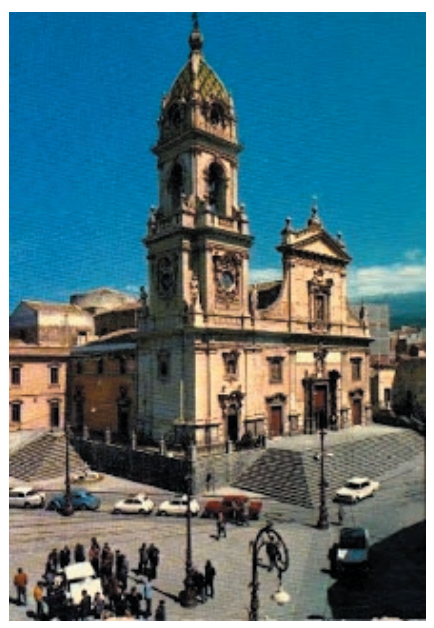

contemporaneo" (Scaccianoce, 2015, p. 22). I biancavillesi "sanno" che la Madonna dell'Elemosina è la loro misericordiosa protettrice, avendolo "dimostrato" più volte nei secoli, a cominciare da quei profughi arbëreshë che si fermarono a Callìcari.

\section{Slancio di fede e devozione mariana durante la pandemia: il caso di Biancavilla}

Come accennato in premessa, in un ideale legame che unisce le generazioni di biancavillesi, anche durante le restrizioni da covid-19, l'attività legata al culto di Maria Elemosina non solo non è venuta meno, ma si è mantenuta costante e ha intensificato alcune attività, servendosi dei mezzi di comunicazione di massa, soprattutto della rete internet, i cui canali sono già ampiamente adoperati dalla locale comunità ecclesiale. Diremmo piuttosto che grazie ai siti internet, alle pagine Facebook ed altri canali social, già prima dell'emergenza da covid-19, la storia e la fede biancavillese hanno potuto varcare lo stretto ambito locale ed essere note a più utenti, anche semplicemente interessati dal punto di vista culturale e non strettamente religioso. ${ }^{13}$ Va detto per completezza che in paese si trovano altre parrocchie, ugualmente attive, ma comunque l'icona bizantina rimane l'elemento agglutinante di tutta la comunità, non soltanto di quella più ristretta della basilica.

\section{Il diario della crisi pandemica a Biancavilla}

5 Marzo 2020: a seguito dell'ordinanza di chiusura delle scuole, l'Arcidiocesi di Catania rende pubbliche le sospensioni delle attività di catechesi, attività pastorali, processioni e tutte le forme di aggregazioni di fedeli. Per la comunità del Santuario di S. Maria dell'Elemosina in Biancavilla inizia così il "tempo del Covid": tutte le attività di catechesi per i ragazzi, i giovani e gli adulti, le attività oratoriali, gli incontri di Azione Cattolica, dell'Associazione "Maria SS. dell'Elemosina", della Caritas parrocchiale, vengono sospese.

Il 7 marzo 2020, tutta la comunità si è radunata in serata per una celebrazione penitenziale, in cui si è implorato la protezione della Beata Vergine Maria, come da sempre il popolo Biancavillese ha fatto nei momenti più disperati (guerre, precedenti pandemie, calamità naturali, ecc).

\footnotetext{
${ }^{13}$ Si rimanda ai seguenti siti internet, che risultano ben curati e di facile consultazione, ricchi anche di una corposa documentazione fotografica, tracce video ed audio: www.santamariaelemosina.it e www.lagrandefestaestiva. it, oltre alle omonime pagine Facebook. Nel 2002 è nata anche l'Associazione omonima, dal 2004 di diritto diocesano, volta a tutelare e promuovere il culto alla Madonna dell'Elemosina. La Confraternita sotto il titolo della Vergine dell'Elemosina e recentemente (2018) anche l'Associazione Culturale Arbëreshë, per la salvaguardia delle radici e tradizioni arbëreshë di Biancavilla e che si cura della divulgazione nelle scuole e promuove manifestazioni culturali e rievocazioni storiche.
} 
Il 9 marzo 2020 inizia il lockdown: vengono sospese le celebrazioni eucaristiche con la presenza dei fedeli. Da questa data in poi, la nostra comunità decide di cambiare rotta, sperimentando un metodo di evangelizzazione diverso, in varie forme, che potesse raggiungere tutti seppur a distanza: le Celebrazioni Eucaristiche domenicali in diretta sui social e attraverso un'emittente televisiva locale; il Prevosto Parroco, don Agrippino Salerno, che quotidianamente inizia a mandare dei brevi videomessaggi di catechesi per dare, ogni giorno, una parola di conforto a tutti; si iniziano a proporre delle attività online per i bambini del catechismo e dell'Oratorio parrocchiale "don Pino Puglisi": ogni sera i giovani, attraverso la diretta Instagram, commentavano il Vangelo del giorno, suonavano e pregavano insieme alle famiglie che si riunite. Nel corso della quaresima, ogni venerdì si è organizzata anche la Via Crucis online in diretta, e anche tutte le celebrazioni della Settimana Santa e della Santa Pasqua sono arrivate nelle tv delle case dei fedeli. Tutti i giorni, attraverso Facebook, l'Associazione "Maria SS. dell'Elemosina" ha promosso una bellissima iniziativa di preghiera: dalle case di tanti fedeli la recita del S. Rosario davanti all'immagine della Madonna dell'Elemosina che ognuno custodisce nella propria abitazione. Liniziativa si è protratta anche nel corso del mese di maggio.

Ma le attività non si sono di certo fermate, anzi, attraverso nuove forme sono andate sempre avanti: i giovani dell'Oratorio si sono dati alla stesura di canti che hanno ottenuto particolari riconoscimenti: "Ama oltre le distanze", un canto di speranza proprio sul periodo che si stava vivendo, e "Maria Cuore di Misericordia", un canto che i giovani hanno voluto dedicare alla Madonna dell'Elemosina. Entrambi i testi hanno ottenuto tante condivisioni e visualizzazioni: Avvenire, VaticanNews, il sito ufficiale della Conferenza Episcopale Italiana, Radio Vaticana, il quotidiano La Sicilia, e molti altri hanno dedicato uno spazio per parlare di questo lavoro "a distanza" dei giovani. Anche il Presidente della Repubblica Italiana, Sergio Mattarella, ha inviato un messaggio per ringraziare del lavoro di speranza promosso durante il lockdown.

Le restrizioni si allentano e, gradatamente, a partire da Maggio 2020, si iniziano a proporre delle attività in sicurezza, come le S. Messe esterne, per i quartieri del territorio, portando la sacra icona della Madonna dell'Elemosina che visitava anziani e ammalati impossibilitati e "impauriti" ad uscire dalle loro case verso la Basilica. Anche la Festa dell'ultima domenica d'Agosto in onore della Madonna dell'Elemosina, preceduta dal tradizionale "novenario" di preparazione, ha subìto una chiara modifica ma non è mancata la devozione di tanti fedeli che sono accorsi, in sicurezza, a rendere omaggio alla protettrice della cittadina. Per ultimo, nel tempo di Natale, è stato allestito un albero natalizio, denominato "della carità", sotto cui i fedeli portavano generi alimentari per gli assistiti della Caritas, sempre più bisognosi di viveri e conforto.

\section{Un primo bilancio della cristocentrica devozione mariana di Biancavilla, tra fede ed impegno civile}

Le indicazioni governative italiane, recepite dalla conferenza episcopale italiana, circa le misure per il contenimento del contagio e le norme di distanziamento sociale, hanno imposto la sospensione pubblica delle celebrazioni liturgiche, durante la gran parte della quaresima e per quasi tutto il periodo pasquale. Per tutto questo lungo periodo, la comunità biancavillese ha seguito con assiduità le celebrazioni tenute in basilica, collegandosi 
regolarmente al sito internet parrocchiale ed alle pagine social, dove erano quotidianamente trasmesse sia la celebrazione eucaristica che la preghiera canonica ed altri momenti di preghiera legati a novene e devozioni locali. Le connessioni alle dirette streaming risultano essere centinaia nel conteggio delle domeniche e feste di precetto, e a decine per le attività e celebrazioni feriali. Questo nonostante l'ampia offerta che le televisioni nazionali hanno ugualmente offerto. ${ }^{14}$ Un dato questo di stretto attaccamento alla propria comunità, sia pur nel necessario isolamento domestico. Di particolare rilevanza ci sembrano tuttavia due ulteriori elementi. Quello dell'attività oratoriale e quello della catechesi. In un'azione pastorale tradizionale, queste attività si sarebbero inevitabilmente fermate o molto rallentate, causando un possibile scollamento dei gruppi e della partecipazione alla vita comunitaria, che non si è fermata nonostante la pandemia sia ugualmente arrivata nel centro etneo, sia pur contenuta e senza gravi conseguenze. Quanto all'attività oratoriale, va rilevata la costante attività online del locale oratorio "don Pino Puglisi". Gli animatori e soprattutto i giovani hanno mantenuto contatti costanti e cospicue attività in streaming, organizzando le attività già programmate per l'estate 2020, che sono infine confluite in un progetto di animazione educativa, pensata per la stagione estiva ed organizzato secondo le indicazioni ministeriali legate alla cosiddetta fase 3 di convivenza con il covid-19. ${ }^{15} \mathrm{Va}$ anche sottolineata l'attività musicale, precipua di ogni oratorio, che ha impegnato i giovani biancavillesi, che hanno composto ed eseguito online, alcuni nuovi inni. ${ }^{16}$ Fra gli altri anche un nuovo inno a Maria Elemosina, ${ }^{17}$ che, pur rinnovandosi nel linguaggio, si situa nel solco della lunga tradizione musicale religiosa biancavillese. Il dato di maggior significato è quello della formazione, più strettamente della catechesi per l'iniziazione cristiana e degli adulti. Essa non è venuta meno, grazie all'impegno degli operatori pastorali, che hanno tenuto i contatti online quanto più fosse possibile non solo con i ragazzi, ma con le famiglie, nell'ottica del nuovo stile comunicativo, definito catecumenale, particolarmente raccomandato da papa Francesco e recepito dalla Chiesa italiana. Questo va detto a fronte di un dato generalmente non confortante, che durante il periodo della pandemia, ha fatto registrare una partecipazione agli eventi online non sempre seguita dappertutto in maniera massiccia e con numeri spesso bassi, come rilevato da uffici catechistici diocesani e recepiti dall'ufficio catechistico nazionale.

\section{Conclusioni}

Quanto alla percezione di una sorta di protezione dalla pandemia, da parte della Vergine dell'Elemosina, è un dato di devozione diffuso nell'animo dei biancavillesi. Né si possono tacciare di superstizione, quanto piuttosto di genuina fede popolare. Né essi sono

\footnotetext{
${ }^{14}$ Sia l'emittente pubblica Rai, sia le reti Mediaset, oltre a Tv2000 della C.E.I. e TelePadrePio. Anche le reti televisive locali hanno offerto agli utenti le celebrazioni liturgiche in streaming, specialmente in occasione della Settimana Santa.

15 "Ama oltre le distanze". Programma consultabile sulla pagina Facebook omonima, post del 13 giugno 2020. https://it-it.facebook.com/oratoriopinopuglisi/photos/a.1825891834388282/2617374705239987/?type=3\&theater consultato 14 giugno 2020.

${ }^{16} \mathrm{Si}$ veda il canale Youtube: https://www.youtube.com/channel/UCgf5wszx7ufqdLxkNr-ZlTw consultato 14 giugno 2020.

17 “Maria, cuore di Misericordia”. Cfr. https://www.youtube.com/watch?v=Z9QsIHB6mfQ consultato 14 giugno 2020.
} 
nuovi a questa ferma convinzione. Anche in passato, come rilevato dagli storici locali, Maria Elemosina avrebbe protetto la comunità biancavillese da calamità naturali, carestie e guerre. ${ }^{18}$ Conta soprattutto il fatto che la comunità biancavillese non si è "disgregata" in tante piccole monadi, ma ha reagito all'emergenza pandemica, legandosi alla sua tradizione religiosa, mantenendo il legame liturgico ed extraliturgico, ma soprattutto rinforzando un'azione catechistico-educativa di ampio respiro che saggiamente "trasmette una dottrina e un sapere (conoscenza della fides quae), ma è anche e soprattutto maturazione di atteggiamenti e assunzione di un progetto di vita (interiorizzazione della fides qua)" (Alberich, 1986, p. 107). ${ }^{19}$ Una fede, dunque, veramente cristiana, che non si lascia sopraffare dal devozionalismo, memore delle tradizioni trasmesse dai padri e conservate in una fedeltà che non scade in mere manifestazioni folcloristiche. La fede dei biancavillesi ha mantenuto viva questa devozione mariana, semplicemente in virtù della sua stessa esistenza, lungo l'arco di questi secoli, senza scambiarla per una sorta di portafortuna o talismano. La positiva esperienza biancavillese insegna che la devozione e la pietà popolare possono - e devono - portare al superiore livello della consapevolezza e della presa di coscienza della fede, espressa anche in corretta forma liturgica. Devozione e pietà popolare che, soprattutto, abbiano un vero carattere performativo, che cioè cambino effettivamente lo stile di vita del fedele. Ancor più va sottolineata l'apertura al dialogo, tipica dell'ambiente biancavillese. $\mathrm{Ma}$ anche per un confronto con altre culture, seppure distanti, almeno in apparenza. Da questo si deve ripartire per approdare ad un concreto senso di apertura e di accoglienza verso l'altro, anche se "lontano" dalla propria cultura d'appartenenza, pur mantenendo doverosamente e comunque vive le proprie radici culturali. È in questottica che va inteso dunque il rapporto vivo ed intenso tra i biancavillesi e la loro icona mariana. In questa immagine, anche nella recente emergenza sanitaria, hanno ritrovato l'essere tutti parte di un'unica comunità, che stringendosi attorno all'icona ha affrontato il pericolo, sentendo la "presenza" mariana viva e vera, pegno e segno di fede che si fa dialogo, che di tutto è la chiave di lettura. Per lortodossia orientale, questo dialogo è fatto di preghiera, di silenzio e di incrocio di sguardi, come quello del bambino e della madre dell'icona biancavillese, che, nel loro guardarsi, in uno rivolgono lo sguardo al fedele che li contempla, il quale è il destinatario di quell'incrocio di sguardi.

\footnotetext{
${ }^{18}$ Si dice che quando Biancavilla è afflitta da siccità o da tempeste tali da minacciare i raccolti, l'intervento della Vergine è sempre puntuale. Nella siccità del 1876 l'icona fu portata in processione con tempo sereno; giunti alla chiesa di S. Orsola, piovve per parecchie ore, salvando il raccolto. Un fatto simile accadde nel maggio del 1948, con testimonianza del prevosto pro tempore Gaetano Messina. Nel 1576 la peste imperversò in Sicilia, e la vicina Paternò ebbe decine di vittime, mentre Biancavilla fu risparmiata, così come avvenne con il colera che colpì duramente la popolazione delle confinanti cittadine di Santa Maria di Licodia e di Adrano nel 1887. Senza contare le numerose eruzioni dell'Etna (specialmente del 1536, 1603, 1669 e, soprattutto del 1879, diretta sul paese, e che deviò inopinatamente, secondo la testimonianza del vulcanologo prof. Orazio Silvestri. Parimenti per i terremoti, compresi quelli, devastanti, del 1693 e del 1883. Durante la seconda guerra mondiale, il 30 giugno 1942, terremoto e colata lavica minacciarono la cittadina; tutto il popolo, con clero ed autorità in testa, portò l'icona sul fronte lavico e questo si arrestò alle ore 21 del 2 luglio successivo. Anche in occasione dei tragici bombardamenti del 1943 il paese fu risparmiato dagli angloamericani, anche in questo caso stranamente, perché esso era cardine dellestreme e disperata difesa italotedesca, che aveva piazzato la contraerea perfino nella centrale piazza Roma. All'improvviso il comando dell'Asse decise di spostare il perno difensivo oltre Bronte, contro ogni previsione, anche degli Alleati, risparmiando così non solo ulteriori lutti e distruzioni al paese (che si ebbero comunque, ma in forma più lieve), ma anche risparmiando dal saccheggio indiscriminato le abitazioni abbandonate da molta parte della popolazione, prudenzialmente rifugiatasi oltre il paese, nella cosiddetta Contrada Vigne (cfr. Monforte, 2008, pp. 131-138).

${ }^{19}$ Cfr. Congregazione per il Culto Divino e la Disciplina dei Sacramenti, (2001).
} 


\section{Bibliografia}

Alberich, E. (1986). Catechesi. In J. Gevaert (a cura di), Dizionario di Catechetica, (pp. 104-108).

Archivio Storico Diocesano dell'Arcidiocesi di Catania, (1507-1509). Fondo Tutt'atti. f. 88-152.

Associazione "Maria SS. dell'Elemosina”, (2015). L'icona di Biancavilla. Nuovi studi e ricerche. Raccolta di documenti e contributi dal $2^{\circ}$ Simposio di studi sulla Vergine dell'Elemosina, Biancavilla (Ct): Author.

Bertanza, G. (2013). La vita vera e autentica è al centro. Luoghi e ritmi sacri per il Sé-ntire nel quotidiano. Trento: Edizioni del Faro.

Bucolo, P. (1953). Storia di Biancavilla. Biancavilla (Ct): Arti Grafiche.

Cilento, A. (2008). Bisanzio in Sicilia e nel Sud dell'Italia. Udine: Magnus.

Congregazione per il Culto Divino e la Disciplina dei Sacramenti, (2001). Direttorio su pietà popolare e Liturgia. Principi e orientamenti, 17 dicembre 2001, in Enchiridion Vaticanum 20, (pp. 2619-2657).

Di Dario Guida, M.P. (2013). Gli eredi di Bisanzio. Le icone postbizantine in Europa e nell'Oriente mediterraneo. In M.K. Guida (ed.), Le icone postbizantine in Sicilia, (pp. 9-55).

Eliade, M., (1968). Discorso pronunciato al Congresso di Storia delle religioni di Boston, 24 giugno 1968. In Bertanza, G., La vita vera e autentica è al centro.

Fondazione Federico II, (2013). Immagine e Scrittura. Presenza greca a Messina dal Medioevo alletà Moderna, Palermo: Author.

Gevaert, J. (a cura di) (1986). Dizionario di Catechetica. Leumann (To): Elle Di Ci.

Gharib, G. (1998). La «Theotokos» nel dialogo ecumenico. Rivista Liturgica, 85, 2-3, 351361.

Giovanni Paolo II, (1981). Decreto per l'istituzione della festa liturgica di S. Maria dell'Elemosina, 31 luglio 1981, in BASP, Cat. IV, Classe I, Fasc. II, n. 67.

Guida, M.K. (a cura di) (2013). Le icone postbizantine in Sicilia. Secoli XV-XVIII. Roma: Gangemi Editore.

Idem, Icone postbizantine nella Sicilia centro-orientale, in Idem (ed.), Le icone postbizantine in Sicilia, (pp. 115-148).

Kalafati, K.PH., Pittori greci in Italia dalla fine del XV secolo al XVIII secolo, Fondazione Federico II, Immagine e Scrittura, (pp. 181-189).

Li Cauli, G. \& Melluso, L. (2015). Storie albanesi di Sicilia. Conversazione con unarbëreshë. "I contesti" 7, Palermo: Istituto Poligrafico Europeo.

Longhitano, A. (2006). Da Callicari a Biancavilla. Lordinamento della cura delle anime. Biancavilla: Biblioteca Comunale "G. Sangiorgio".

Mandalà, M. (2015). Biancavilla nel contesto delle migrazioni albanesi del sec. XV. In Associazione "Maria SS. dell'Elemosina”, L’icona di Biancavilla, (pp. 33-38).

Mellusi, G.G., (2013). La Chiesa greca di Messina e nel Valdemone (secoli XII-XVI). Giurisdizione e controversie. In Fondazione Federico II, Immagine e Scrittura, (pp. 265280).

Monforte, A., (2008). Il culto di Maria SS. dell'Elemosina a Biancavilla tra storia, fede e pietà popolare. Biancavilla (Ct): Biblioteca Comunale "G. Sangiorgio". 
Pasku, K. (2015). Gli italo-albanesi: dalle migrazioni alle tradizioni culturali e religiose. In Associazione "Maria SS. dell'Elemosina”, L’icona di Biancavilla, (pp. 39-45).

Perroy, E. (1958). Storia generale delle civiltà. III. Il Medioevo. Firenze: Casini.

Piccione, F. (2004). La porta d'Oriente. La fondazione della Chiesa Annunziata, primo segno di rinnovamento spirituale e culturale a Biancavilla all'alba del Seicento, Biancavilla: Biblioteca Comunale "G. Sangiorgio".

Rodriquez, M.T., Cultura greca, libri e biblioteche, in Fondazione Federico II, Immagine e Scrittura, (pp. 217-250).

Salerno, A., (2015). Una storia di cui essere orgogliosi, in Associazione "Maria SS. dell'Elemosina", L'icona di Biancavilla, (pp. 6-7).

Sangiorgio, P.A., (2015). Storia per silenzi di un viaggio e di un approdo, in Associazione "Maria SS. dell'Elemosina", L'icona di Biancavilla, (pp. 10-16).

Santangelo, G., (2015), Un legame d'affetto inseparabile, in Associazione "Maria SS. dell'Elemosina”, L'icona di Biancavilla, (pp. 8-9).

Scaccianoce, A., (2015). Il "prodigio del fico": un mito che rilegge la storia con gli occhi di Dio. In Associazione "Maria SS. dell'Elemosina”, L’icona di Biancavilla, (pp. 17-22).

Schiavone, A., (2015). La tecnica iconografica della Vergine di Biancavilla: i suoi prototipi e i criteri per una datazione, in Associazione "Maria SS. dell'Elemosina", L'icona di Biancavilla, (pp. 23-28).

Velmans, T. (2013). Larte dell'icona. Storia, stile, iconografia dal V al XV secolo. Milano: Jaca Book.

\section{Sitografia}

www.santamariaelemosina.it

www.lagrandefestaestiva.it

https://it.facebook.com/oratoriopinopuglisi/photos/a.1825891834388282/26173747052399

$87 /$ ?type $=3 \&$ theater

https://www.youtube.com/channel/UCgf5wszx7ufqdLxkNr-ZlTw

https://www.youtube.com/watch?v=Z9QsIHB6mfQ 